
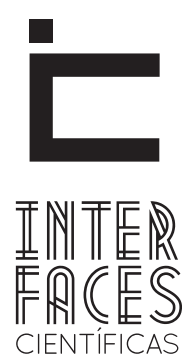

SAÚDE E AMBIENTE

ISSN IMPRESSO 2316-3313

ISSN ELETRÔNICO 2316-3798

\title{
CÂNCER PEDIÁTRICO: SOBREVIDA EM SERGIPE-BRASIL, 1980-2004
}

\author{
Margareth Rose Uchoa Rangel ${ }^{1}$ \\ Enaldo Vieira Melo ${ }^{3}$ \\ Carlos Anselmo Lima ${ }^{5}$
}

\author{
Amaury Lelis Dal Fabbro ${ }^{2}$ \\ Arthur Rangel Azevedo ${ }^{4}$ \\ Rosana Cipolotti ${ }^{6}$
}

\section{RESUMO}

O câncer pediátrico é a segunda causa de morte em crianças e adolescentes. As taxas de sobrevida são indicadores importantes para o conhecimento da qualidade dos cuidados oferecidos a essa população. Analisar a sobrevida de pacientes de zero a 19 anos portadores de neoplasias malignas, acompanhados em dois serviços hospitalares de oncologia de Aracaju, Sergipe. Estudo descritivo, realizado com informações dos registros hospitalares dos Serviços de Oncologia Pediátrica de Aracaju e do Sistema de Mortalidade do Estado de Sergipe. Selecionados 1203 casos entre 1980 a 2004. As taxas de sobrevidas foram estimadas por meio do método de Kaplan Meier. Resultados: a sobrevida global estimada em cinco anos dos pacientes estudados foi de $46,4 \%$. Os pacientes portadores de neoplasias epiteliais, nas neoplasias germinativas, trofoblásticas e gonadais e neoplasias do sistema nervoso central, apresentaram as maiores taxas de sobrevidas. Os pacientes com sarcomas de partes moles e leucemias apresentaram as menores taxas. A comparação das sobrevidas nos dois serviços incluídos mostrou diferenças significativas. As taxas de sobrevida aumentaram progressivamente de $35,9 \%$ no período de $1980-1984$ para $54,7 \%$ no período de $1995-1999$ e caiu para $50,6 \%$ no período de 2000-2004. De maneira geral, ao longo dos anos observou-se melhora significativa nas taxas de sobrevida, embora tenha havido um decréscimo no último período estudado.

\section{PALAVRAS CHAVES}

Sobrevida. Câncer. Crianças. Adolescentes. 


\section{ABSTRACT}

Pediatric cancer is the second leading cause of death in children and adolescents, and its survival rates are important indicators of the health care quality offered to this population. Objectives: Analyze the survival rate of patients with malignant neoplasms from zero to 19 years old treated in two cancer hospitals in Aracaju. Method: A descriptive study based on data from hospital records of Pediatric Oncology Services of Aracaju and Mortality Rate System of the State of Sergipe. For this study, 1203 cases from 1980 to 2004 were selected. The survival rates were estimated using the Kaplan-Meier method. Results: The estimated overall five years survival was $46.4 \%$. Patients with epithelial, germ cell, trophoblastic, gonadal and central nervous system neoplasms had the highest survival rates. Patients with soft tissue sarcomas and leukemia had the lowest rates. A comparison of the two services included survival showed significant differences. Survival rates increased progressively from $35.9 \%$ during $1980-1984$ to $54.7 \%$ in the period $1995-$ 1999 , however it fell to $50.6 \%$ in $2000-2004$. Conclusions: Overall, there was significant improvement in survival rates over the years, although there was a decrease in the final period.

\section{KEY WORDS}

Survivorship. Cancer. Children. Teenagers.

\section{RESUMEN}

El cáncer pediátrico es la segunda causa de muerte en niños y adolescentes. Las tasas de supervivencia son indicadores importantes para el conocimiento de la calidad de la atención ofrecida a esa población. Objetivos: analizar la supervivencia de pacientes de Zero a 19 años con neoplasia maligna, seguidos en dos servicios hospitales de oncología de Aracaju, Sergipe. Método: estudio descriptivo, realizado con informaciones de los registros hospitalarios de los Servicios de Oncología Pediátrica de Aracaju y del Sistema de Mortalidad del Estado de Sergipe. Fueron seleccionados 1203 casos desde 1980 hasta 2004. Las tasas de supervivencia fueron estimadas a través del método Kaplan Meier. Resultados: la supervivencia global estimada en cinco años de los pacientes estudiados fue de 46,4\%. Los pacientes con neoplasias epiteliales, en neoplasias germinativas, trofoblasticas y gonadales y neoplasias del sistema nervioso central presentaron las mayores tasas de supervivencia. Los pacientes con sarcoma de tejidos blandos y leucemias presentaron las menores tasas. La comparación de las supervivencias en los dos servicios inclusos mostró diferencias significativas. Las tasas de supervivencia aumentaron progresivamente de $35,9 \%$, en el periodo de 1980 1984 , para $54,7 \%$, en el periodo de $1995-1999$, y se ha reducido para 50,6\% en el periodo de 2000-2004. Conclusiones: de manera general, a través de los años se observó una mejora significativa en las tasas de supervivencia, aunque hubo una disminución en el último periodo de estudios.

\section{PALABRAS CLAVE}

Supervivencia. Cáncer. Niños. Adolescentes. 


\section{INTRODUÇ̃̃̃O}

O câncer pediátrico corresponde a 1 - 3\% de todos os tumores malignos. Nos últimos 50 anos a sobrevida global de crianças com diagnóstico de câncer passou de $10 \%$ para $80 \%$ ou mais. As taxas de sobrevida são indicadores importantes para o conhecimento da qualidade dos cuidados oferecidos às crianças com câncer, entretanto as estatísticas sobre as neoplasias pediátricas no Brasil têm sido pouco analisadas na literatura médica nacional.

\section{MÉTODOS}

O estado de Sergipe está localizado na região nordeste do Brasil, sua população é de 2.068.031 habitantes, sendo $36,74 \%$ de zero a 19 anos. Possui dois serviços de referência para o tratamento de doenças oncológicas: A Fundação Beneficente Hospital de Cirurgia (FBHC) e Centro de Oncologia Dr. Osvaldo Leite do Hospital de Urgência de Sergipe Governador João Alves Filho (HGJAF). Foram analisados os dados de 1203 pacientes oncológicos, de zero a 19 anos, cadastrados nos dois serviços de oncologia no período de 1980 a 2004 e dados do Sistema de Informação de Mortalidade do Estado de Sergipe.
Em Sergipe foram realizados dois estudos epidemiológicos sobre o câncer pediátrico, utilizando os registros hospitalares dos dois serviços de oncologia do estado, incluindo 895 casos no período de 1980-1999 e 255 casos no período de 2000-2004; o percentual de óbitos encontrado foi de $52,7 \%$ e $45,9 \%$ respectivamente.

O objetivo deste trabalho foi estimar a sobrevida global de pacientes pediátricos oncológicos, matriculados nos serviços de oncologia pediátrica de Sergipe.

Trata-se de um estudo descritivo. Os dados foram analisados por meio de testes estatísticos, considerando o intervalo de confiança de $95 \%$, para o teste de Student e qui-quadrado, respectivamente para análise das variáveis quantitativas e categóricas. A técnica de Kaplan-Meier, do programa estatístico SPSS versão 19.0, foi utilizada para estimar a sobrevida, sendo estabelecido o número mínimo de vinte casos, para realização dessa análise, para maior confiabilidade do resultado. 


\section{RESULTADOSE DISCUSSÃO}

Entre os anos de 1980 e 2004 foram diagnosticados 1203 casos de neoplasias malignas em crianças e adolescentes, entre zero e dezenove anos de idade, em Sergipe, sendo 727 casos $(60,4 \%)$ matriculados na FBHC.
Observou-se predomínio dos pacientes não-brancos com 818 casos (68,0\%), refletindo as características demográficas da população de Sergipe. Predominaram os pacientes do gênero masculino, com 724 casos $(60,2 \%)$. Os linfomas e as leucemias (tumores hematológicos) juntos somaram 651 casos (54,1\%), conforme tabela 1 abaixo.

Tabela 1 - Pacientes oncológicos pediátricos dos serviços de oncologia pediátrica de Aracaju-Sergipe de 1980-2004

\begin{tabular}{llrrlrrr}
\hline DISTRIBUIÇÃo & GERAL & $\mathbf{N}$ & $\mathbf{\%}$ & & $\mathbf{N}$ & $\mathbf{\%}$ & TOTAL \\
Serviços & FBHC* & 727 & 60,4 & HGJAF** & 476 & 39,6 & 1203 \\
Estado & Sergipe & 1072 & 89,1 & Outros & 131 & 10,9 & 1203 \\
Cidades & Aracaju & 344 & 28,6 & Outras & 728 & 71,4 & 1203 \\
Etnia & Brancos & 385 & 32,0 & Outros & 818 & 68,0 & 1203 \\
Gênero & Masculino & 724 & 60,2 & Feminino & 479 & 39,8 & 1203 \\
Tipo de tumor & Sólidos & 552 & 45,9 & Hematológicos & 651 & 54,15 & 1203 \\
\hline
\end{tabular}

Fonte: pesquisa *Fundação Beneficente Hospital de Cirurgia; ** Hospital

Governador João Alves Filho.

O grupo morfológico mais frequente foi o grupo das leucemias com 331 casos (27,5\%; IC 25,3$30,1 \%)$, seguido pelo grupo dos linfomas e neoplasias do sistema retículo endotelial (NRE), com 320 casos (26,6\%; IC 24,2-29,3\%), as neoplasias ósseas com 95 casos (7,9\%; IC 6,4-9,5\%), os tumores renais com 94 casos (7,8\%; IC 6,3-9,4\%), as neoplasias do sistema nervoso central (SNC) com 85 casos (7,1\%, IC 5,7-8,6\%), as neoplasias do sistema nervoso simpático (SNS) com 77 casos (6,4\%; IC 5,1-7,7\%), o grupo dos sarcomas de partes moles com 67 casos (5,6\%; IC 4,4-6,8\%), o retinoblastoma com 51 casos ( $4,2 \%$; IC 3,2-5,3\%), as neoplasias epiteliais com 37 casos (3,1\%; IC 2,2$4,1 \%)$, as neoplasias germinativas trofoblásticas e gonadais com 35 casos (2,9\%; IC 2,0-3,9\%) e por fim as neoplasias hepáticas com 10 casos (1\%; IC 0,3-1,3\%).
Em todas as faixas etárias houve predomínio dos tumores hematológicos sobre os tumores sólidos. As neoplasias renais, do sistema nervoso simpático, retinoblastoma, neoplasias hepáticas, os tumores do sistema nervoso central, os sarcomas de partes moles, além das leucemias e linfomas, foram mais frequentes no grupo etário de zero a quatro anos. No grupo de cinco aos nove anos os linfomas foram mais frequentes, seguidos pelas leucemias e tumores do SNC. No grupo dos 10 aos 14 anos observou-se maior frequência dos tumores de células germinativas e trofoblásticas e principalmente dos tumores ósseos. No grupo dos maiores de quinze anos predominaram os linfomas, leucemias e as neoplasias ósseas. Os carcinomas e as neoplasias epiteliais, extremamente raros nos menores de cinco anos, aumentaram em frequência com a idade, sendo mais frequente entre os $10 \mathrm{e}$ 14 anos, conforme tabela 2 abaixo: 
Tabela 2 - Distribuição dos tumores por grupo morfológico x grupo etário em Sergipe, de 1980 a 2004

\begin{tabular}{|c|c|c|c|c|c|c|c|c|c|c|}
\hline Grupo etário & $0 \mathrm{a}$ & & 5 a & & 10 a 14 & & 15 a 19 & & Total & \\
\hline Grupo Morfológico & $\mathrm{N}$ & $\%$ & $\mathrm{~N}$ & $\%$ & $\mathrm{~N}$ & $\%$ & N & $\%$ & & $\%$ \\
\hline Linfomas e NRE & 113 & 9,4 & 102 & 8,5 & 65 & 5,4 & 40 & 3,3 & 320 & 26,6 \\
\hline Neop.germ,trofo e gon & 8 & 0,7 & 9 & 0,7 & 12 & 1 & 6 & 0,5 & 35 & 2,9 \\
\hline Neoplasias do SNS & 57 & 4,7 & 15 & 1,2 & 3 & 0,2 & 2 & 0,2 & 77 & 6,4 \\
\hline Neoplasias do SNC & 31 & 2,6 & 29 & 2,4 & 18 & 1,5 & 7 & 0,6 & 85 & 7,1 \\
\hline Neoplasias epiteliais & 4 & 0,3 & 8 & 0,7 & 17 & 1,4 & 8 & 0,7 & 37 & 3,1 \\
\hline Neoplasias ósseas & 5 & 0,4 & 16 & 1,3 & 45 & 3,7 & 29 & 2,4 & 95 & 7,9 \\
\hline Neoplasias renais & 70 & 5,8 & 19 & 1,6 & 4 & 0,3 & 1 & 0,1 & 94 & 7,8 \\
\hline Retinoblastoma & 47 & 3,9 & 2 & 0,2 & 1 & 0,1 & 1 & 0,1 & 51 & 4,2 \\
\hline Sarcoma partes moles & 32 & 2,7 & 22 & 1,8 & 8 & 0,7 & 5 & 0,4 & 67 & 5,6 \\
\hline Neoplasias hepáticas & 8 & 0,7 & 1 & 0,1 & 0 & 0 & 1 & 0,1 & 10 & 0,8 \\
\hline Não especificadas & 1 & 0,1 & 0 & 0,0 & 0 & 0,0 & 0 & 0,0 & 1 & 0,1 \\
\hline TOTAL & 508 & 42,2 & 313 & 26 & 255 & 21,2 & 127 & 10,6 & 1203 & 100 \\
\hline
\end{tabular}

N, Número de casos. NSRE, Neoplasias do Sistema Reticuloendotelial. Neop germ trof e gon, Neoplasias germinativas, trofoblásticas e gonadais. SNC, Sistema Nervoso Central. SNS, Sistema Nervoso Simpático.

Ao longo dos anos aumentou o número de diagnósticos de câncer nas crianças e adolescentes de Sergipe, passando de 149 casos no período de 1980-1984 para 316 no período de 2000-2004, sugerindo maior acesso aos serviços de saúde e melhor atenção por parte de médicos e equipes de saúde em reconhecer sinais e sintomas que possibilitem o diagnóstico do câncer na criança e no adolescente. E observou-se que a frequência de abandono de tratamento passou de $23,2 \%$ (IC 12,1-23,5\%) no período de $1990-1994$ para 3,5\% (IC $1,6-5,1 \%)$ no período de 2000-2004 ( $<<0,0001$ ), refletindo melhor adesão ao tratamento oncológico.

A sobrevida global em cinco anos foi de 46,4\% (IC 43,4$49,4 \%)$. Nos serviços de oncologia analisados observam-se problemas financeiros, estruturais e sociais importantes que levam ao atraso no diagnóstico e à descontinuidade do tratamento e pode-se considerar que alguns fatores estejam influenciando nos resultados negativos encontrados nos serviços estudados (RANGEL, et al, 2006).

As taxas de sobrevida em cinco anos, calculadas no Canadá (82\%), Europa (75\%) e Estados Unidos (79\%), são exemplos disso (GATTA, et al, 2005); (ELLISON, POGANY, MERY, 2007).

Apesar dos grandes avanços tecnológicos e científicos na abordagem do câncer pediátrico, nos países em desenvolvimento, a probabilidade de sobrevida é muito baixa, podendo não passar dos 20 a $25 \%$ em algumas localidades do leste europeu, 37,5\% em Bangalore, na Índia, 37\% em Namíbia, na África; 48\% em Cuba e $48 \%$ em Goiânia, no Brasil Braga PE (LATORRE, CURADO, 2002); (LACOUR, et al, 2000). 
Observou-se sobrevida de 50,2\% (IC 44,4-56,0\%) no grupo de 5-9 anos, no grupo dos 0-4 anos sobrevida de 45,9\% (IC 41,3-50,5\%), no de 10-14 de 45,1\% (IC $38,7-51,5 \%$ ) e no grupo dos $15-19$ anos $41,7 \%$ (IC $32,1-50,1 \%)(p=0,154)$.

Os pacientes do serviço de oncologia da $\mathrm{FBHC}$ tiveram 42,1\% (IC 38,3-45,9\%) de sobrevida, enquanto que os do HGJAF 52,9\% (IC 58,3-67-5\%) $(p<0,001)$. Isso provavelmente se deve por que em 1996 foi criado o Serviço de Oncologia do HGJAF, com um serviço estruturado e com uma equipe multidisciplinar qualificada e estimulada, classificado como CACON I (Centro de Alta Complexidade em Oncologia).

Nos pacientes de Aracaju a sobrevida foi de $49,4 \%$ (IC $43,8-55,0 \%$ ) e nos pacientes das outras cidades foi de $45,1 \%$ (IC 41,5-48,7\%) ( $p=0,137)$. 0 gênero masculino obteve maior sobrevida (48,2\%; IC 44,4-52,0\%) que o feminino (43,3\%; IC 44,4$52,0 \%)(p=0,102)$. Os pacientes com tumores sólidos tiveram sobrevida de $47,1 \%$ (IC 43,1-50,1\%) enquanto os hematológicos de $45,8 \%$ (IC 41,8$49,8 \%)(p=0,108)$.

Analisando a estimativa de sobrevida global em cinco anos ao longo do tempo, agrupando por períodos de cinco anos, observou-se que no período de 1980-1984 a sobrevida foi de 36,9\% (IC 28,944,9\%), aumentou para 39,3\% (IC 31,7-46,9\%) no período de 1985-1989, para 42,5\% (IC 36,3$48,7 \%)$ no período de 1990-1994, atingiu o maior percentual no período de 1995-1999 (54,7\%; IC 48,5-60,9\%) decrescendo para 50,6\% (IC 45,0$56,2 \%)$ no período de 2000-2004, observou-se significância estatística $(p>0,001)$ quando as taxas são analisadas em conjunto. Entretanto não existe significância estatística quando analisados isoladamente os dois últimos períodos, conforme figura 1 ao lado:
Figura 1 - Sobrevida dos pacientes oncológicos pediátricos por período de seguimento, Aracaju, Sergipe, 1980 a 2004

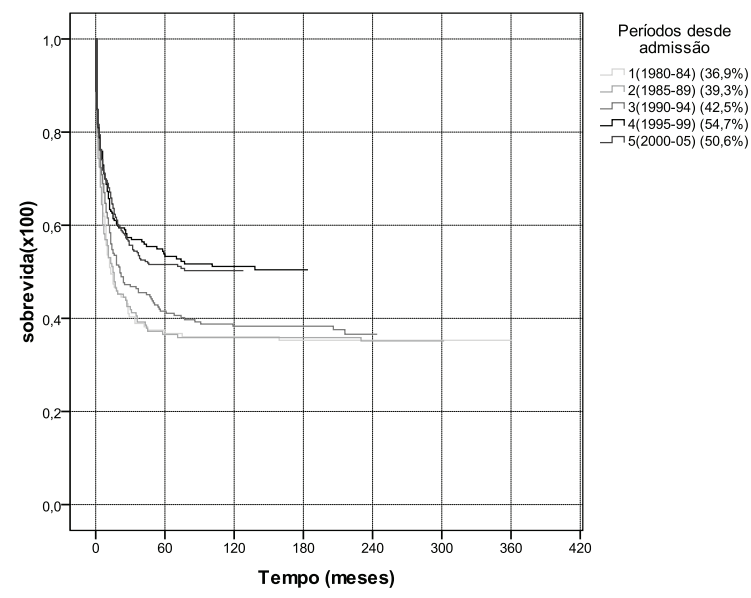

Ao longo dos anos a sobrevida dos pacientes pediátricos portadores de câncer vem aumentando, passando de $10 \%$ nos anos de 1950 para $80 \%$ nos dias atuais, graças à interação entre estratégias de diagnóstico mais precoce e progressos no tratamento. Avanços técnicos nos exames de imagem permitiram diagnósticos mais acurados, técnicas moleculares que identificam anormalidades genéticas, identificação precoce de recaídas ou falhas terapêuticas, além de novos protocolos mais eficientes e menos tóxicos.

No Canadá a sobrevida em cinco anos passou de $71 \%$ de 1994 para 83\% em 2003, na Inglaterra passou de $42 \%$ no período de $1968-1977$ para $71 \%$ no período de 1988-1995 $5^{13}$, nos EUA aumentou de $63 \%$ no período de $1975-1979$ para $79 \%$ no período de 1995 $1999^{15}$, na Europa nos anos de 1970, a sobrevida em cinco anos que era de $44 \%$, nos anos 80 passou para $64 \%$, atingindo $74 \%$ nos anos 90 . Analisando-se dados dos anos de 1995-2002 a probabilidade de sobrevida em cinco anos na Europa foi de $81 \%$, similar à dos Estados Unidos (KAATSCH, 2010). 
Observou-se diferença de sobrevida em cinco anos segundo os grupos morfológicos $(p=0,004)$. O grupo que apresentou maior sobrevida foi o dos carcinomas e neoplasias malignas epiteliais com $62,2 \%$ (IC 46,0-78,4\%), seguido pelo grupo das neoplasias germinativas, trofoblásticas e gonadais com $57,1 \%$ (IC 39,9-74,3\%), neoplasias do SNC com 55,4\% (IC 43,9-66,7\%) e o grupo dos linfomas com $51,9 \%(46,1-57,7 \%)$. As menores sobrevidas foram observadas no grupo dos sarcomas de partes moles com 38,8\% (IC 26,4-51,2\%), leucemias com $39,9 \%$ (IC 34,3-45,5\%) e neoplasias do SNS (40,3\%, IC 28,6-51,2\%).
Quando analisada a sobrevida por subtipos morfológicos conforme a Classificação Internacional do Câncer em Crianças (ICCC) observa-se que, excluídos aqueles dados cuja frequência foi inferior a 20 casos por patologia, as maiores sobrevidas em cinco anos foram observadas no grupo das neoplasias do sistema retículo endotelial (78,4\%; IC 64,0-92,8\%), astrocitomas (75,5\%; IC 57,0-93,0\%) e linfomas de Hodgkin (69,5\%; IC 59,1-79,9\%). As menores taxas de sobrevida em cinco anos foram observadas no grupo das leucemias mielóides agudas (15,6\%; IC 3,2-28,0\%), rabdomiossarcomas (26,5\%; IC 13,4-39,9\%) e no linfoma de Burkitt (35,7\%; IC 20,3-51,1\%), de acordo com a tabela 3, a seguir:

Tabela 3 - Sobrevida dos pacientes oncológicos de Sergipe, por subtipos morfológicos, 1980-2004

\begin{tabular}{|c|c|c|c|c|c|}
\hline GRUPOS MORFOLÓGICOS & $\mathbf{N}$ & Óbitos & Vivos & $\begin{array}{r}\text { Sobre } \\
\% \\
\end{array}$ & IC $95 \%$ \\
\hline I- Leucemias & 331 & 199 & 132 & 39,9 & $34,3-45,5$ \\
\hline la- LLA & 287 & 166 & 121 & 42,2 & $36,2-42,2$ \\
\hline Ib- LMA & 32 & 27 & 5 & 15,6 & $3,2-28,0$ \\
\hline IC- LMC & 8 & 3 & 5 & - & - \\
\hline Id-Outras leucemias & 4 & 3 & 1 & - & - \\
\hline II- Linfomas e NRE & 320 & 154 & 166 & 51,9 & $46,1-57,7$ \\
\hline Ila- Linfoma de Hodgkin & 82 & 25 & 57 & 69,5 & $59,1-79,9$ \\
\hline Ilb- Linfomas não Hodgkin & 159 & 94 & 65 & 40,9 & $32,0-48,9$ \\
\hline Ilc- Linfoma de Burkitti & 42 & 27 & 15 & 35,7 & $20,3-51,1$ \\
\hline Ild- NRE & 37 & 8 & 29 & 78,4 & $64,0-92,8$ \\
\hline III- Tumores do SNC & 85 & 38 & 47 & 55,3 & $43,9-66,7$ \\
\hline llla- Ependimomas & 14 & 9 & 5 & - & - \\
\hline lllb- Astrocitomas & 28 & 7 & 21 & 75,5 & $57,0-93,0$ \\
\hline Illc- Tumor neuroectodermico & 10 & 5 & 5 & - & - \\
\hline Illd-Outros Gliomas & 14 & 6 & 8 & - & - \\
\hline
\end{tabular}




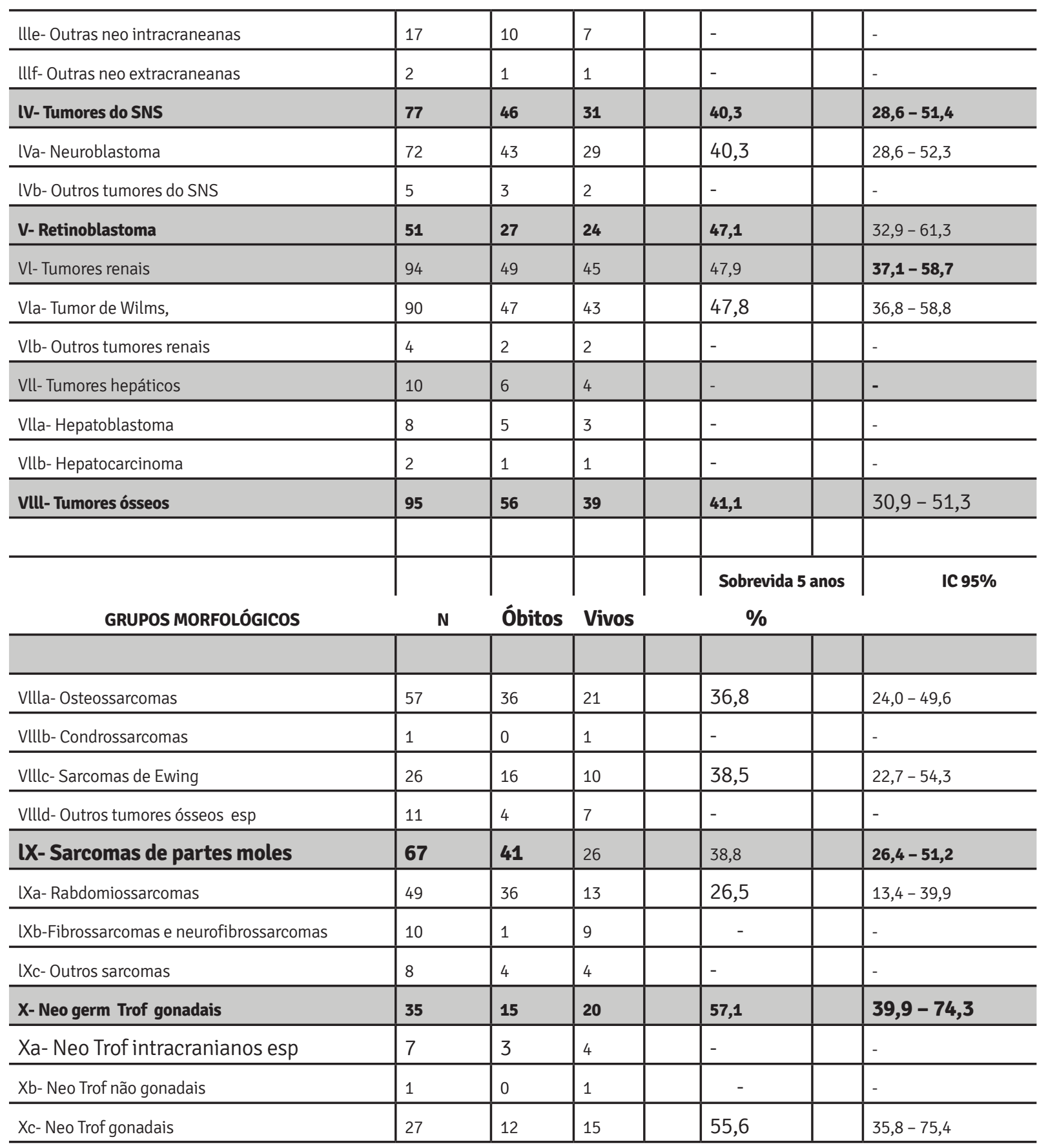




\begin{tabular}{|c|c|c|c|c|c|}
\hline $\mathrm{Xl}$ - Carcinomas e outros tu epiteliais & 37 & 14 & 23 & 62,2 & $46,0-78,4$ \\
\hline Xlb- Carcinoma de Tireóide & 6 & 0 & 6 & - & - \\
\hline Xlc- Carcinoma de nasofaringe & 14 & 9 & 5 & - & - \\
\hline Xld- Melanoma Malígno & 3 & 1 & 2 & - & - \\
\hline Xle- Carcinoma de pele & 11 & 2 & 9 & - & - \\
\hline Xlf- Outras neo epiteliais & 3 & 2 & 1 & - & - \\
\hline Xll- Outras Neop não especificadas & 1 & $\mathbf{0}$ & 1 & - & - \\
\hline TOTAL & 1203 & 645 & 558 & 46,4 & $43,4-49,4$ \\
\hline
\end{tabular}

N- número de casos; IC- intervalo de confiança; LLA- leucemia linfoide aguda; LMA- leucemia mieloide aguda; LMC- leucemia mieloide crônica; NER- neoplasia retículo-endotelial; SNC- sistema nervoso central; SNS- sistema nervoso simpático; Esp- especificada; Neo- neoplasias; germ- germinativas; trof- trofoblásticas; Tu- tumores.

Diversos fatores são considerados determinantes no prognóstico e na sobrevida dos pacientes pediátricos oncológicos, como: idade e estadiamento ao diagnóstico, tipo histológico, perfil imunológico e genético entre outros. (DAMA, et al, 2006). Os tumores do SNC costumam ter o prognóstico reservado e a sobrevida baixa.

Na União Europeia a sobrevida global em cinco anos foi de $61 \%$ e de $69 \%$ no Canadá. Nos EUA no período de 1973-2007 foi de 51,8\%. No presente estudo os tumores do SNC representaram apenas $7,1 \%$ dos casos (sugerindo sub-registro de casos) e apresentou a terceira maior taxa de sobrevida em cinco anos $(55,4 \%)$. É possível que crianças portadoras de tumores menos agressivos tenham sido diagnosticadas, tratadas e acompanhadas nos serviços, enquanto que, pacientes com doença muito agressiva ou por falta de acesso a métodos diagnósticos, como tomografia (só disponível no Estado de Sergipe a partir do ano de 1991) e ressonância magnética (disponível em 2000), tenham morrido antes de se chegar ao diagnóstico, consequentemente não foram matriculados nos serviços de oncologia, tendo como resultado uma sobrevida "falsamente" elevada nesse grupo de tumores.
O grupo das leucemias, que tem sobrevida em cinco anos de $82,1 \%$ nos EUA (Viana, et al,2003) no Canadá de $82 \%$ (Ellison, et al, 2007), em Sergipe foi o grupo de segunda menor sobrevida em cinco anos (39,9\%). Nos países em desenvolvimento, assim como na maioria dos estados brasileiros estudados, a sobrevida em cinco anos no grupo das leucemias costuma ser baixa: $31 \%$ em Belo Horizonte; $48 \%$ em Goiânia; 49\% em Fortaleza (FERREIRA, 2010); (BRA$\mathrm{GA}$, et al, 2007).

Pacientes pediátricos com leucemias agudas requerem protocolos quimioterápicos agressivos e frequentemente necessitam de tratamento de suporte intensivo, e em localidades cujos centros de oncologia estão mais bem estruturados, observa-se maior sobrevida, como em Recife com $63 \%$ e São Paulo com 70\% (BRANDALISE, ONCOL, 2007). O retinoblastoma é o tumor pediátrico de maior sobrevida em cinco anos, chegando a $100 \%$ na Nova Zelândia, $99 \%$ no Canadá, $91 \%$ na União Europeia e $90 \%$ nos EUA (GATTA et al, 2005); (ELLISON, et al, 2007); (LACOUR, et al, 2005). Em Sergipe a sobrevida por retinoblastoma foi de apenas $47,1 \%$ e isso se deve possivelmente ao diagnóstico tardio da doença. 


\section{CONCLUSÕES}

A probabilidade de sobrevida global em cinco anos dos pacientes estudados foi de $46,4 \%$. As maiores sobrevidas foram observadas nas neoplasias epiteliais, nas neoplasias germinativas, trofoblásticas e gonadais e neoplasias do sistema nervoso central. As menores sobrevidas nos sarcomas de partes moles, leucemias e neuroblastomas.

\section{REFERÊNCIAS}

ADAMS, H.; AVENDAÑO, J.; RAZA, S. M.; GOKASLAN, Z. L.; JALLO, G.; QUIÑONES-HINOJOSA, A. Prognostic Factors and Survival in Primary Malignant Astrocytomas of the Spinal Cord: A Population-Based Analysis from 1973 to 2007. Spine (Phila Pa 1976), v.37 (12): 727-735, 2012.

BOSCHMONAR MG, ALVAREZ YG, GARCIA AM, SOTO TN, ROGER MC. Childhood cancer survival in Cuba. Eur J Epidemiol, 16(8): 763-7, 2000.

BRAGA PE, LATORRE MRD, CURADO MP. Childhood cancer: a comparative analysis of incidence, mortality, and survival in Goiania (Brazil) and other countries.

Cad Saúde Publica. 18(1): 33-44, 2002.

BRANDALISE S. Comparison of intermittent versus contínuos methotrexate plus 6-MP in maintenance regimen for standard risk acute lynfoblastic leukemia in children (GBTLI ALL-99). J Clin Oncol, 25(Suppl 18): S9512, 2007.

CHA ES, KONG KA, MOON EK, KHANG YH, LEE WJ. Childhood cancer mortality and birth characteristics in Korea: A national population-basead birth cohort study.J. Koran Med Sci, 26: 339-45, 2011.
Os pacientes tratados no HGJAF tiveram sobrevida significativamente maior que os da FBHC.

De maneira geral, ao longo dos anos do estudo, observou-se melhora significativa nas taxas de sobrevida, embora tenha havido um decréscimo no último período estudado.

DAMA E, PASTORE G, MOSSO ML, MAULE MM, ZUCCOFO L. Late deaths among five-year survivors of childhood cancer. A population-based study in Piedmont Region, Italy. Haematologica, 91(8): 1084-91, 2006.

ELLISON LF, POGANY L, MERY LS. Childhood and adolescent cancer survival: a period analysis of data from the Canadian Cancer Registry. Eur J Cancer, 43(13): 1967-75, 2007.

FERREIRA JMO. Incidência, mortalidade e sobrevida de leucemia e linfoma no município de Fortaleza, Ceará [tese]. Rio de Janeiro: Fundação Osvaldo Cruz, 2010.

GATTA, G, CAPOCACCIA R, COLEMAN MP, RIES GLA, BERRINO F. Childhood cancer survival in Europe and the United States. Cancer, 95(8): 1767-72, 2002.

GATTA G, CAPACACCIA R, STILLER C, KAATSCH P, TERENZIANI M. Childhood cancer survival trends in Europe: a EUROCARE Working Group study. J Clin Oncol, 2005.

IBGE, 2010. Disponível em: <www.ibge.gov.br/.../populacao/censo2010/tabelas_pdf/total_populacao_ sergipe.pdf. Acesso em: 20/03/2013 
INCA, SOBOPE. Câncer na criança e no adolescente no Brasil: dados dos Registros de Base Polulacional e de mortalidade. Ed. INCA, Ministério da Saúde, Sociedade Brasileira de Oncologia Pediátrica. Rio de Janeiro: Gráfica Esdeva, 2008.

HOLNER MJ, RIES LAG, KRAPCHO M, NEYMAN N, AMINOU R. SEER Cancer Statistic Review, 19752006. Nacional cancer Institute. Bethesda, MD, based on November 2008 SEER data submission, posted to the SEER web site, 2009. Disponível em: <http://seer. cancer.gov/csr/1975_2006/>. sergipe.pdf. Acesso em: $22 / 03 / 2013$

KAATSCH P. Epidemiology of childood cancer. Cancer Treatment Review, 36: 277-85, 2010.

LACOUR B, DESANDES E, MALLOL N, SOMMELET D. LORRAINE. Childhood cancer registry: incidence, survival 1983-1999. Arch Pediatr, 12(11): 1577-86, 2005.

MISSAOUI N, KHOUZEMI M, LANDOLSI H, JAIDENE L, ABDELKRIM SB et al. Childhood cancer frequency in the Center of Tunísia. Asian Pacific Journal of Cancer Prevention, 12: 537-42, 2011.

O'LEARY M, KRAILO M, ANDERSON JR, REAMAN GH. Progress in childhood cancer: 50 years of research collaboration, a report from the Children's Oncology Group. Semin Oncol. 35(5): 484-93, 2008.

PEDROSA MF, PEDROSA F, LINS MM, PONTES NETO NT, HANOIS FILHO G. Non-Hodgkin's lymphoma in childhood: clinical and epidemiological characteristics and survival analysis at a single center in Northe- ast Brazil. J Pediatr (Rio J), 83(6): 547-54, 2007.

RANGEL MRU, CIPOLOTTI R, FABBRO AL, FONTES AM, BOTELHO M. Estudo epidemiológico do câncer em menores de vinte anos no estado de Sergipe-Brasil, no período de 1980-1999. Revista Brasileira de Cancerologia, 48(2): 271-276, 2002.

RANGEL MRU, SANTOS KSSJ, CIPOLOTTI R., FONTES AM. Epidemiologia do Câncer Pediátrico no Estado de Sergipe-Brasil. Atualização e Estudo Comparativo. SIICSalud 2006; 1-9 DOI. Disponível em: <http:// www.siicsalud.com/dat049/06802015.htm>. sergipe. pdf. Acesso em: 20/03/2013

RIES LAG, EISNER MP, KOSARY CL, HANKEY BF, MILLIERBA, CLEGG L. SEER Cancer statistic review, 1973-1997 ( monography on the internet). Bethesda: National Cancer Institute; 2002 [cited 2008 out 2]. Available from: Disponível em: <http://seer.cancer.gov/csr/1973_1997/>. sergipe.pdf. Acesso em: 25/03/2013

STELIAROVA-FOUCHER, E, STILLER C, KAATSCH P, BERRINO F COEBERG JW. Geographical patterns and time trends of cancer incidence and survival among children and adolescents in Europe since the 1970s (the ACCISproject): an epidemiological study. Lancet, 364(9451): 2097-105, 2004.

VIANA MB, CUNHA KCCMS, RAMOS G., MOURÃO M. Acute myeloide leukemia in childhood: fifteen-year experience in a single instituition. J Pediatr (Rio J), 79(6): 489-96, 2003. 
1 Possui graduação em Medicina pela Universidade Federal da Paraíba (1986), Residência Médica no Hospital Pedro Ernesto da UERJ (19871989), Pós-graduação em cirurgia pediátrica oncológica no INCA (19901991), Mestrado em Saúde da Criança pela Universidade Federal de Sergipe (2000). Doutora em Medicina em Ciências da Saúde pela Universidade Federal de Sergipe (2013). E-mail: mrrangel@uol.com.br

2 Doutor em Medicina Preventiva pela Faculdade de Medicina de Ribeirão Preto da Universidade de São Paulo, Brasil (1997). Trabalha no Conselho Nacional de Desenvolvimento Científico e Tecnológico, Brasil. E-mail: adfabbro@frmp.usp.br

3 Possui graduação em Medicina pela Universidade Federal de Sergipe (1997), Mestrado em Ciências Médicas pela Faculdade de Medicina de Ribeirão Preto (2003) e residência-médica pela Universidade Federal de Sergipe (1999). Atualmente é da Universidade Federal de Sergipe. E-mail: evmsidarta@hotmail.com

4 Possui graduação em Medicina pela Universidade Federal de Sergipe. E-mail: rangel.aaz@gmail.com

5 Possui graduação em Medicina pela Universidade Federal de Sergipe (1985), Residência Médica em Cancerologia com área de atuação em Cirurgia Oncológica (1989), Mestrado em Desenvolvimento e Meio Ambiente pela Universidade Federal de Sergipe (2000). Doutor em Ciências da Saúde pela Universidade Federal de Sergipe (2013). E-mail: limaca@infonet.com

6 Possui graduação em Medicina pela Faculdade de Medicina de Ribeirão Preto-Universidade de São Paulo (1982), Mestrado em Medicina-Pediatria (1990). Doutora em Medicina-Oncologia Pediátrica (2000) pela Faculdade de Medicina de Ribeirão Preto-Universidade de São Paulo. Especialista em Cancerologia (área de atuação: Oncologia Pediátrica) pela Associação Médica Brasileira e Sociedades Brasileiras de Cancerologia e Oncologia Pediátrica. E-mail: rosanaci@yahoo.com 